\title{
WEBAPPENDIX
}

\section{Cost effectiveness of improved hypertension management in India through increased treatment coverage and adherence: a mathematical modelling study}

\author{
Technical Details \\ - Microsimulation Model \\ - Probability of Clinical Events \\ - Modelling the coverage of hypertension treatment \\ - Modelling the treatment adherence to hypertension treatment \\ - Costs \\ Results \\ - Model Validation \\ - Cost and health outcomes for Overall Population \\ - Health outcomes for Females \\ - Health outcomes for Males \\ - One-way sensitivity analysis \\ Reporting Checklists \\ - Consolidated Health Economic Evaluation Reporting Standards (CHEERS) checklist
}

\section{Tables}

Webappendix Table S1: Illustration of current and next health states in the model Webappendix Table S2: Age and sex-specific monthly probability of non-CVD death Webappendix Table S3: Age and sex-specific probability of MI and Stroke upon CVD event Webappendix Table S4: Age and sex-specific thirty-day probability of death after myocardial infarction and stroke

Webappendix Table S5: Age and sex-specific monthly probability of death for non-fatal myocardial infarction and stroke

Webappendix Table S6: Implementation of the treatment protocol

Webappendix Table S7: Age and SBP-specific relative risk of MI and Stroke due to antihypertensive medication

Webappendix Table S8: Cost of anti-hypertensive medication disaggregated by public sector and retail prices Webappendix Table S9: Cost of CVD care medication disaggregated by public sector and retail prices Webappendix Table S10: Programmatic cost of an IHCI-like program Webappendix Table S11: Costs and health outcomes associated with the population of 40 to 69yr from 2020-40 across different coverage and adherence scenarios

Webappendix Table S12: Simulated health outcomes for Females between 40-69yr across different coverage and adherence scenarios

Webappendix Table S13: Simulated health outcomes for Males between 40-69yr across different coverage and adherence scenarios

Webappendix Table S14: One-way sensitivity analysis for costs and health outcomes associated with the population of 40 to $69 \mathrm{yr}$ from $2020-40$ for the $70 \%$ coverage and adherence scenario

Webappendix Table S15: Simulated health outcomes for Females between 40-69yr across one-way sensitivity analysis

Webappendix Table S16: Simulated health outcomes for Males between 40-69yr across one-way sensitivity analysis

Webappendix Table S17: Consolidated Health Economic Evaluation Reporting Standards (CHEERS) checklist

\section{Figures}

Webappendix Figure S1: Schematic of Model Structure

Webappendix Figure S2: Validation of model output versus GBD 2017 estimates 


\section{Microsimulation Model}

2 We developed a discrete-time microsimulation model to simulate individual progression through health states

3 for a hypothetical cohort comprising of 10,000 individuals between 40 to 69 year old without history of CVD

4 event (Well state) at the start of the simulation. The model follows each individual in one-month cycles for a

5 simulation period of 20 years.

7 We simulated the model for two cohorts of 10,000 individuals each for males and females, respectively, with

8 individual profiles sampled from the WHO SAGE Wave 1 dataset for India.[1] We ensured (i) the proportion of

9 individuals in age brackets of 40-44, 45-49, 50-54, 55-59, 60-64, 65-69 in our simulated cohort was equal to the

10 observed demographic characteristics for India, and (ii) the prevalence of hypertension in age- and sex-specific

11 brackets in our simulated cohort was equal to the original dataset. We did not add individuals turning 40 years

12 old in the subsequent cycles into the cohort.

14 In each cycle, an individual in the Well state can experience one of three possible clinical events and associated

15 state transition: (i) no CVD event and thus stays in the current Well state (ii) occurrence of a CVD event and transitions to the CVD Event state, (iii) non-CVD related death and transitions to the terminal Death state.

18 The CVD event state comprises of an occurrence of either myocardial infarction (MI) or stroke. Upon entering

19 the transitory CVD Event state, an individual experiences two possible clinical events: (i) a fatal CVD event, and

20 thus moves to the terminal Death state, or (ii) a non-fatal CVD event and moves to either the postMI or

21 postStroke chronic state. An individual in the postMI or postStroke state can experience one of three clinical events: (i) recurrence of a CVD event, and thus transition to CVD Event state, (ii) death and thus, move to the terminal Death state, or (iii) no CVD event, and stay in the current postMI or postStroke state. The structure of the model is provided in figure S1, along with the state transitions in table S1.

\section{Probability of Clinical Events}

\section{Risk of a non-CVD Death}

28 We retrieved the probability of all-cause deaths for India from WHO's Global Heath Data repository.[2]

29 Further, we calculated the proportion of non-CVD deaths to all-cause deaths from GBD 2017 across 2012-

30 17.[3] We were thus able to determine the monthly probability of non-CVD deaths in India (Table S2). 


\section{Risk of a CVD Event}

33 We determined the risk of a CVD event using the Globorisk office calculator.[4] The inputs for the calculator

34 include an individual's age, sex, systolic blood pressure, smoking habit and BMI. These values are available

35 from the WHO SAGE Wave I dataset. The calculator provides 10-yr probability of a CVD event, and we

36 converted it to a monthly probability assuming a constant hazard over the 10-year period.[5] We calculated the

37 risk of a CVD event for each individual in the model every five years to incorporate the effect of increase in age

38 and systolic blood pressure. The age-related increase in systolic blood pressure has been modelled based on a

39 study by Bellows et al.[6] We calculated the conditional probability of either MI or stroke upon a CVD event

40 (Table S3) based on the observed ratio of incidence of MI to stroke in the GBD 2017 study across 2012-17.[3]

\section{Risk of death post CVD event}

43 A CVD event could lead to either fatality within the first month (fatal CVD event), or the individual surviving

44 for the first month (non-fatal CVD event). In case of a non-fatal CVD event, the individual experiences a higher

45 risk of death compared to the risk of all-cause death among individuals in Well state. We calibrated the values

46 for risk of death post MI or stroke such that the model estimated mortality matches the observed mortality of

47 death in 2017 from the GBD 2017 study. The model validation result is presented in Figure S2, and the

48 calibrated monthly probability of death due to MI and stroke in Table S4 and S5.

49

\section{$50 \quad$ Risk of an additional CVD event}

51 The monthly probability of a CVD event during the first month after an MI is based on the control arm in ACS

52 QUIK study in Kerala, India.[7] The 30-day probability of repeat stroke during the first month is determined

53 from the findings of Petty et al.[8] If an individual experiences a non-fatal MI or Stroke, the risk of an additional

54 CVD event is based on a study by Lin et al.[9] The relative risk of death after an additional CVD event is 1.5

55 compared to risk of death after the first CVD event.[10]

Relative risk of CVD due to treatment

58 We determined the required drug dosage for which the individual's blood pressure decreases below 140mmHg

59 based on the study by Law et al.[11] Based on the required dosage, we assigned specific combination of drugs

60 for each individual based on the treatment protocol (Table S6). The treatment protocol is based on the approved 
61 IHCI protocol being implemented in the state of Punjab currently.[12] Based on the combination of drugs

62 administered, the initial blood pressure and the age of the individual, we determined the relative risk of MI and

63 stroke from the study by Law et al (Table S7).

\section{Modelling Coverage of Hypertension Treatment}

65 The coverage of hypertension program was calculated as the ratio of hypertensive individuals on treatment to the total number of hypertensive individuals. We modeled the coverage of treatment by modifying two components: (i) the proportion of hypertensive individuals who were aware of their blood pressure, and (ii) the proportion of status-aware individuals initiated on treatment. To implement the first component, in each simulation run, we allocated individuals to be either aware of their blood pressure or not. The proportion of status-aware individuals in status-quo (i.e. current coverage of treatment in India) was based on findings of the Prospective Urban Rural Epidemiology Study (PURE) study.[13] For the second component, we first determined if the individuals, who were aware of their blood pressure, were also eligible for hypertensive treatment based on either the NPCDCS guideline (in status quo), or the simplified protocol (in intervention arm). $[14,15]$ Further, among the eligible individuals, only a proportion of individuals were initiated on treatment. The treatment initiation ratio for the status quo was also based on the PURE study.[13] Based on the two components, we find $17 \%$ of the population to be covered by hypertension treatment in the status-quo, and we apply CVD risk reduction only to individuals who are initiated on the treatment.

\section{Modelling Adherence to Hypertension Treatment}

The adherence to treatment is calculated as the ratio of hypertensive individuals highly adherent to medication to the total number of individuals initiated on treatment. We modeled the adherence to treatment by modifying two components: (i) the proportion of individuals who persist with treatment for more than a year, (ii) the proportion of individuals who comply with the prescribed medication dosage and interval over the duration of their treatment. We implement the first component by assigning each individual either as persistent or nonpersistent (discontinues treatment after an year). We used the results from a study by Van Wijk et al[16] for proportion of individuals persistent with treatment due to paucity of India-specific data. We assume that individuals who persist with treatment after one year neither drop out of treatment nor consume less than $50 \%$ of the prescribed medication.[16] In order to implement medication compliance among persistent individuals, we assign individuals to be either highly adherent or moderately adherent. Highly adherent individuals consume more than $80 \%$ of the pills on time and according to prescribed dosage. Moderately adherent individuals 
consume between 50 to $80 \%$ of the pills on time and according to prescribed dosage. The proportion of highly

91 adherent to medication is based on a study in India by Dennis et al.[17] The individuals who are highly adherent

92 to medication experience the complete effect of the risk reduction, whereas individuals who are moderately

93 adherent to medication experience only $50 \%$ of the risk reduction due to medication. This is based on a previous

94 modelling study by Cherry et al.[18] Individuals who discontinue treatment after a year do not experience any

95 reduction in risk of CVD.

\section{Costs}

97 We used data from the government contracts in the state of Telangana to determine the cost of antihypertensive

98 drugs in the government sector.[19] We assumed a 30\% overhead cost on the contract rates to account for

99 logistics and inventory management of the drug.[20] The costs of drugs in the private sector was calculated by

100 taking the mean selling price of the first five brand of drugs suggested by the Indian online pharmacy,

101 1mg.com.[21] The medication cost per individual was calculated by adding the cost of specific drug molecules

102 in the individual's treatment regimen and their dosage for the individual. The costs have been provided in Table

103 S8. For individuals moderately adherent on medication, we varied the costs between $50 \%$ and $80 \%$ (based on a

104 normal distribution) of the fully adherent medication cost.

106 We assumed monthly doctor consultations for the first six months of treatment, followed by a quarterly

107 consultation. The cost of doctor consultation was taken from a study by Prinja et al on community health centres

108 and primary health centres across four states in India.[22] We included a one-time diagnostic test cost based on

109 central government health scheme rate card.[23] The programmatic cost of a hypothetical intervention based on

110 the tenets of IHCI was determined through discussions with experts and has been expanded in Table S10. The

111 programmatic costs include the salaries of additional human resources, the cost of training the medical staff, and

112 the operating costs for running the tech platform. We the required resources and their respective cost was based

113 on IHCI's implementation in nine districts of Telangana.

115 The cost of acute CVD care comprised of consultation, hospital room costs, procedures, and medication cost

116 and the cost of chronic CVD care comprised of outpatient consultation cost and chronic medication cost. The

117 values were based on data from WHO-CHOICE,[24] and calculated by a previous cost-effectiveness study by

118 Lin et al.[9] Further, we included the cost of percutaneous coronary intervention (PCI) and coronary artery

119 bypass graft(CABG) among 4.2\% and 1.7\% of the patients with acute MI.[25] All individuals with chronic 
120 CVD incurred CVD medication cost. The cost of chronic CVD medication in the government sector was

121 determined from the median buyer price in the International Drug Price Indicator.[26] The cost of CVD

122 medication cost in private sector was based on the mean selling price of the first five brands suggested by

123 1mg.com.[21] The costs have been provided in Table S9. All costs were scaled to the year 2020 with an

124 inflation factor of $3 \%$ per annum. 
Webappendix Table S1: Illustration of current and next health states in the model

\begin{tabular}{|c|c|c|}
\hline$\#$ & Current State & Possible Next State(s) \\
\hline \multirow{3}{*}{1} & \multirow{3}{*}{ Well } & Well \\
\hline & & CVD Event \\
\hline & & Death \\
\hline \multirow{3}{*}{2} & \multirow{3}{*}{ CVD Event } & postMI \\
\hline & & postStroke \\
\hline & & Death \\
\hline \multirow{3}{*}{3} & \multirow{3}{*}{ postMI } & postMI \\
\hline & & CVD Event \\
\hline & & Death \\
\hline \multirow{3}{*}{4} & \multirow{3}{*}{ postStroke } & postStroke \\
\hline & & CVD Event \\
\hline & & Death \\
\hline 5 & Death & Death \\
\hline
\end{tabular}

Webappendix Table S2: Age and sex-specific monthly probability of non-CVD death

\begin{tabular}{|l|c|c|}
\hline Age & Female* & Male* \\
\hline $40-44$ & $0.000206-0.000210$ & $0.000324-0.000330$ \\
\hline $45-49$ & $0.000257-0.000263$ & $0.000427-0.000434$ \\
\hline $50-54$ & $0.000381-0.000389$ & $0.000568-0.000579$ \\
\hline $55-59$ & $0.000544-0.000554$ & $0.000834-0.000849$ \\
\hline $60-64$ & $0.001013-0.001026$ & $0.001224-0.001245$ \\
\hline $65-69$ & $0.001658-0.001679$ & $0.002029-0.002058$ \\
\hline $70-74$ & $0.002744-0.002773$ & $0.003114-0.003153$ \\
\hline $75-79$ & $0.004026-0.004072$ & $0.004574-0.004626$ \\
\hline $80-84$ & $0.006411-0.006512$ & $0.006525-0.006648$ \\
\hline $85+$ & $0.011137-0.011312$ & $0.011201-0.011413$ \\
\hline
\end{tabular}

* The ranges provided are 95\% CI for monthly probability of death due to all causes except CVDs, with the values sampled based on a $\beta$ distribution in the simulation runs.

Webappendix Table S3: Age and sex-specific proportion of MI to Stroke upon CVD event

\begin{tabular}{|l|c|c|}
\hline Age & Female* & Male* \\
\hline $40-44$ & $0.375-0.378$ & $0.435-0.444$ \\
\hline $45-49$ & $0.384-0.386$ & $0.471-0.484$ \\
\hline $50-54$ & $0.418-0.424$ & $0.507-0.527$ \\
\hline $55-59$ & $0.451-0.461$ & $0.534-0.560$ \\
\hline $60-64$ & $0.497-0.510$ & $0.562-0.589$ \\
\hline $65-69$ & $0.536-0.551$ & $0.587-0.611$ \\
\hline $70-74$ & $0.557-0.574$ & $0.625-0.647$ \\
\hline $75-79$ & $0.569-0.589$ & $0.668-0.691$ \\
\hline $80-84$ & $0.560-0.574$ & $0.660-0.677$ \\
\hline $85+$ & $0.560-0.574$ & $0.660-0.677$ \\
\hline
\end{tabular}

* The ranges provided are $95 \%$ CI for ratio of MI to Stroke occurrence upon a CVD event, with the values sampled based on a $\beta$ distribution in the simulation runs. 
Webappendix Table S4: Age and sex-specific thirty-day probability of death after myocardial infarction and stroke

\begin{tabular}{|c|c|c|c|c|}
\hline \multirow[t]{2}{*}{ Age } & \multicolumn{2}{|c|}{ Female } & \multicolumn{2}{|c|}{ Male } \\
\hline & MI & Stroke & MI & Stroke \\
\hline $40-44$ & 0.0132 & \multirow{9}{*}{0.1188} & 0.0330 & \multirow{9}{*}{0.1262} \\
\hline $45-49$ & 0.0132 & & 0.0330 & \\
\hline $50-54$ & 0.0132 & & 0.0330 & \\
\hline $55-59$ & 0.0188 & & 0.0470 & \\
\hline $60-64$ & 0.0188 & & 0.0470 & \\
\hline $65-69$ & 0.0356 & & 0.0890 & \\
\hline $70-74$ & 0.0356 & & 0.0890 & \\
\hline $75-79$ & 0.0516 & & 0.1290 & \\
\hline $80+$ & 0.0516 & & 0.1290 & \\
\hline
\end{tabular}

Webappendix Table S5: Age and sex-specific monthly probability of death for non-fatal myocardial infarction and stroke

\begin{tabular}{|l|c|c|c|c|}
\hline \multirow{2}{*}{ Age } & \multicolumn{2}{|c|}{ Female } & \multicolumn{2}{c|}{ Male } \\
\cline { 2 - 5 } & MI & Stroke & MI & 0.00499 \\
\hline $40-44$ & 0.000525 & 0.000541 & 0.005487 & 0.001699 \\
\hline $45-49$ & 0.001981 & 0.000916 & 0.005786 & 0.002398 \\
\hline $50-54$ & 0.002155 & 0.001456 & 0.006085 & 0.003196 \\
\hline $55-59$ & 0.002562 & 0.001622 & 0.006085 & 0.003894 \\
\hline $60-64$ & 0.002969 & 0.001747 & 0.006980 & 0.005288 \\
\hline $65-69$ & 0.003317 & 0.002120 & 0.008470 & 0.006582 \\
\hline $70-74$ & 0.003491 & 0.002494 & 0.009760 & 0.008371 \\
\hline $75-79$ & 0.003897 & 0.002991 & 0.019342 & 0.012534 \\
\hline
\end{tabular}


Webappendix Table S6: Implementation of the treatment protocol

\begin{tabular}{|c|l|c|}
\hline $\begin{array}{c}\text { Initial Systolic } \\
\text { Blood Pressure } \\
(\mathrm{mmHg})\end{array}$ & \multicolumn{1}{|c|}{ Medication Administered } & $\begin{array}{c}\text { Estimated reduction in systolic } \\
\text { blood pressure (mmHg) }\end{array}$ \\
\hline 140 & Amlodipine 5mg & 5.9 \\
\hline 150 & Amlodipine 10mg & 8.7 \\
\hline 150 & Amlodipine 10mg + Telmisartan 40mg & 15.4 \\
\hline 160 & Amlodipine 10mg + Telmisartan 80mg & 18.4 \\
\hline 160 & Amlodipine 10mg + Telmisartan 80mg + Chlorthalidone 12.5mg & 25.9 \\
\hline 170 & Amlodipine 10mg + Telmisartan 80mg + Chlorthalidone 25mg & 29 \\
\hline 180 & Amlodipine 10mg + Telmisartan 80mg + Chlorthalidone 25mg & 31.7 \\
\hline
\end{tabular}

Webappendix Table S7: Age and SBP-specific relative risk of MI and Stroke due to antihypertensive medication

\begin{tabular}{|c|c|c|c|}
\hline \multirow{2}{*}{ Age } & \multirow{2}{*}{$\begin{array}{l}\text { Initial SBP } \\
\text { (in } \mathrm{mmHg} \text { ) }\end{array}$} & \multicolumn{2}{|c|}{ Relative Risk } \\
\hline & & MI & ST \\
\hline \multirow[t]{5}{*}{$40-49$} & 140 & 0.810 & 0.740 \\
\hline & 150 & 0.675 & 0.575 \\
\hline & 160 & 0.485 & 0.355 \\
\hline & 170 & 0.390 & 0.255 \\
\hline & $180+$ & 0.320 & 0.200 \\
\hline \multirow[t]{5}{*}{$50-59$} & 140 & 0.810 & 0.750 \\
\hline & 150 & 0.685 & 0.595 \\
\hline & 160 & 0.495 & 0.375 \\
\hline & 170 & 0.400 & 0.275 \\
\hline & $180+$ & 0.330 & 0.220 \\
\hline \multirow[t]{5}{*}{$60-69$} & 140 & 0.830 & 0.780 \\
\hline & 150 & 0.710 & 0.630 \\
\hline & 160 & 0.535 & 0.425 \\
\hline & 170 & 0.440 & 0.320 \\
\hline & $180+$ & 0.380 & 0.260 \\
\hline \multirow[t]{5}{*}{$70-79$} & 140 & 0.860 & 0.890 \\
\hline & 150 & 0.755 & 0.805 \\
\hline & 160 & 0.595 & 0.665 \\
\hline & 170 & 0.505 & 0.585 \\
\hline & $180+$ & 0.440 & 0.530 \\
\hline \multirow[t]{5}{*}{$80+$} & 140 & 0.890 & 0.890 \\
\hline & 150 & 0.805 & 0.805 \\
\hline & 160 & 0.665 & 0.665 \\
\hline & 170 & 0.585 & 0.585 \\
\hline & $180+$ & 0.530 & 0.530 \\
\hline
\end{tabular}


Webappendix Table S8: Cost of anti-hypertensive medication disaggregated by public sector and retail prices

\begin{tabular}{|l|c|c|}
\hline \multirow{2}{*}{ Drug } & \multicolumn{2}{|c|}{ Cost per pill } \\
\cline { 2 - 3 } & Public Sector (INR) & Retail (INR) \\
\hline Amlodipine 5mg & 0.169 & 1.04 \\
\hline Telmisartan 40mg & 0.767 & 2.72 \\
\hline Chlorthalidone $12.5 \mathrm{mg}$ & 0.780 & 8.24 \\
\hline
\end{tabular}

Webappendix Table S9: Cost of CVD care medication disaggregated by public sector and retail prices

\begin{tabular}{|l|c|c|}
\hline \multirow{2}{*}{ Drug } & \multicolumn{2}{|c|}{ Cost per pill } \\
\cline { 2 - 3 } & Public Sector (INR) & Retail (INR) \\
\hline Aspirin $75 \mathrm{mg}$ & 0.98 & 0.28 \\
\hline Atenolol $50 \mathrm{mg}$ & 0.721 & 1.75 \\
\hline Lisinopril $10 \mathrm{mg}$ & 9.38 & 12.40 \\
\hline Simvastatin $20 \mathrm{mg}$ & 3.717 & 14.55 \\
\hline
\end{tabular}

Webappendix Table S10: Programmatic cost of an IHCI-like program

\begin{tabular}{|c|c|c|}
\hline Parameter & & Value \\
\hline Number of districts served & & 9 \\
\hline Population covered & & 9545455 \\
\hline Proportion of population greater than $40 \mathrm{yr}$ & & $27 \%$ \\
\hline Population serviced by the intervention & & 2577273 \\
\hline Cost Entities & Number of entities & Cost per entity (INR) \\
\hline Cardiovascular Health Officer & 4 & 150,000 per month \\
\hline State Treatment Supervisor & 9 & 31,000 per month \\
\hline Training sessions per district per annum & 12 & 100,000 per session \\
\hline Technology teams allocated to a state & 1 & 100,000 per month \\
\hline Platform infrastructure & 1 & 100,000 per annum \\
\hline Annual Cost of Intervention (INR) & & $2,26,48,000$ \\
\hline Cost of Intervention per individual serviced (INR) & & 8.79 \\
\hline Cost of Intervention per individual serviced (US \$) & & 0.13 \\
\hline
\end{tabular}


Webappendix Table S11: Costs and health outcomes associated with the population of 40 to $69 y$ from $2020-40$ across different coverage and adherence scenarios

\begin{tabular}{|c|c|c|c|c|c|c|c|c|}
\hline \multirow[b]{2}{*}{ Coverage } & \multirow[b]{2}{*}{ Adherence } & \multirow[b]{2}{*}{$\begin{array}{c}\text { ICER } \\
\text { (\$/DALY averted) }\end{array}$} & \multirow[b]{2}{*}{$\begin{array}{l}\text { DALYs Averted } \\
\text { (percent, 95\% UI) }\end{array}$} & \multirow[b]{2}{*}{$\begin{array}{l}\text { Incremental Cost } \\
\text { (percent, 95\% UI) }\end{array}$} & \multicolumn{2}{|c|}{$\begin{array}{l}\text { Per-capita incremental } \\
\text { costs over } 20 \text { years }\end{array}$} & \multirow{2}{*}{$\begin{array}{c}\text { Annual net } \\
\text { expenditure } \\
\text { (in '000 US } \\
\$ \text { ) }\end{array}$} & \multirow{2}{*}{$\begin{array}{c}\text { Probability of } \\
\text { Cost Saving } \\
\text { (percent) }\end{array}$} \\
\hline & & & & & $\begin{array}{c}\text { Antihypertensive Program } \\
(\$, 95 \% \text { UI })\end{array}$ & $\begin{array}{l}\text { CVD Treatment } \\
(\$, 95 \% \text { UI })\end{array}$ & & \\
\hline 0.4 & 0.4 & 350.89 & $\begin{array}{c}0.347 \\
\text { (0.342 to } 0.353)\end{array}$ & $\begin{array}{c}0.72 \\
(0.7 \text { to } 0.73)\end{array}$ & $\begin{array}{c}6.62 \\
(6.59 \text { to } 6.65)\end{array}$ & $\begin{array}{c}-3.88 \\
(-3.94 \text { to }-3.83)\end{array}$ & 52649 & 0.004 \\
\hline 0.4 & 0.6 & 88.74 & $\begin{array}{c}0.528 \\
(0.522 \text { to } 0.535)\end{array}$ & $\begin{array}{c}0.28 \\
(0.26 \text { to } 0.29)\end{array}$ & $\begin{array}{c}7.09 \\
(7.06 \text { to } 7.12)\end{array}$ & $\begin{array}{c}-6.04 \\
(-6.11 \text { to }-5.97)\end{array}$ & 20255 & 0.172 \\
\hline 0.4 & 0.8 & 8.85 & $\begin{array}{c}0.877 \\
(0.869 \text { to } 0.885)\end{array}$ & $\begin{array}{c}0.05 \\
(0.02 \text { to } 0.07)\end{array}$ & $\begin{array}{c}10.28 \\
\text { (10.23 to } 10.33)\end{array}$ & $\begin{array}{c}-10.11 \\
(-10.19 \text { to }-10.03)\end{array}$ & 3354 & 0.451 \\
\hline 0.4 & 1 & Cost Saving & $\begin{array}{c}1.141 \\
(1.132 \text { to } 1.15)\end{array}$ & $\begin{array}{c}-0.28 \\
(-0.3 \text { to }-0.25)\end{array}$ & $\begin{array}{c}12.1 \\
\text { (12.04 to } 12.16)\end{array}$ & $\begin{array}{c}-13.16 \\
(-13.25 \text { to }-13.07)\end{array}$ & -20392 & 0.737 \\
\hline 0.6 & 0.4 & 141.10 & $\begin{array}{c}0.821 \\
(0.811 \text { to } 0.83)\end{array}$ & $\begin{array}{c}0.68 \\
(0.65 \text { to } 0.71) \\
\end{array}$ & $\begin{array}{c}11.83 \\
\text { (11.77 to } 11.9)\end{array}$ & $\begin{array}{c}-9.23 \\
(-9.34 \text { to }-9.13)\end{array}$ & 50020 & 0.078 \\
\hline 0.6 & 0.6 & 3.97 & $\begin{array}{c}1.091 \\
\text { (1.081 to } 1.101)\end{array}$ & $\begin{array}{c}0.03 \\
(-0.01 \text { to } 0.06)\end{array}$ & $\begin{array}{c}12.54 \\
\text { (12.48 to } 12.61)\end{array}$ & $\begin{array}{c}-12.45 \\
(-12.56 \text { to }-12.34)\end{array}$ & 1870 & 0.478 \\
\hline 0.6 & 0.8 & Cost Saving & $\begin{array}{c}1.615 \\
\text { (1.603 to } 1.627)\end{array}$ & $\begin{array}{c}-0.31 \\
(-0.35 \text { to }-0.27)\end{array}$ & $\begin{array}{c}17.32 \\
(17.23 \text { to } 17.41)\end{array}$ & $\begin{array}{c}-18.51 \\
(-18.63 \text { to }-18.39)\end{array}$ & -22881 & 0.695 \\
\hline 0.6 & 1 & Cost Saving & $\begin{array}{c}2.015 \\
\text { (2.002 to } 2.027)\end{array}$ & $\begin{array}{c}-0.82 \\
(-0.86 \text { to }-0.78)\end{array}$ & $\begin{array}{c}20.05 \\
\text { (19.95 to } 20.15)\end{array}$ & $\begin{array}{c}-23.18 \\
(-23.3 \text { to }-23.05)\end{array}$ & -60101 & 0.897 \\
\hline 0.7 & 0.7 & Cost Saving & $\begin{array}{c}1.68 \\
\text { (1.668 to } 1.692)\end{array}$ & $\begin{array}{c}-0.36 \\
(-0.4 \text { to }-0.33)\end{array}$ & $\begin{array}{c}18.04 \\
\text { (17.95 to } 18.13)\end{array}$ & $\begin{array}{c}-19.43 \\
(-19.55 \text { to }-19.3)\end{array}$ & -26741 & 0.721 \\
\hline 0.8 & 0.4 & 72.08 & $\begin{array}{c}1.301 \\
(1.29 \text { to } 1.313)\end{array}$ & $\begin{array}{c}0.55 \\
(0.51 \text { to } 0.59)\end{array}$ & $\begin{array}{c}17.04 \\
\text { (16.95 to } 17.13)\end{array}$ & $\begin{array}{c}-14.94 \\
(-15.05 \text { to }-14.82)\end{array}$ & 40519 & 0.174 \\
\hline 0.8 & 0.6 & Cost Saving & $\begin{array}{c}1.661 \\
\text { (1.649 to } 1.673)\end{array}$ & $\begin{array}{c}-0.31 \\
(-0.35 \text { to }-0.27)\end{array}$ & $\begin{array}{c}17.98 \\
\text { (17.89 to } 18.08)\end{array}$ & $\begin{array}{c}-19.17 \\
(-19.29 \text { to }-19.04)\end{array}$ & -22785 & 0.688 \\
\hline 0.8 & 0.8 & Cost Saving & $\begin{array}{c}2.352 \\
(2.338 \text { to } 2.365)\end{array}$ & $\begin{array}{c}-0.76 \\
(-0.81 \text { to }-0.72)\end{array}$ & $\begin{array}{c}24.34 \\
(24.21 \text { to } 24.46)\end{array}$ & $\begin{array}{c}-27.26 \\
(-27.39 \text { to }-27.12)\end{array}$ & -56155 & 0.857 \\
\hline 0.8 & 1 & Cost Saving & $\begin{array}{c}2.879 \\
\text { (2.865 to } 2.894)\end{array}$ & $\begin{array}{c}-1.42 \\
(-1.47 \text { to }-1.37)\end{array}$ & $\begin{array}{c}27.98 \\
\text { (27.84 to } 28.12)\end{array}$ & $\begin{array}{c}-33.4 \\
(-33.54 \text { to }-33.26)\end{array}$ & -104243 & 0.957 \\
\hline 1 & 0.4 & 47.30 & $\begin{array}{c}1.77 \\
\text { (1.758 to } 1.782)\end{array}$ & $\begin{array}{c}0.49 \\
(0.45 \text { to } 0.53)\end{array}$ & $\begin{array}{c}22.24 \\
\text { (22.12 to } 22.37 \text { ) }\end{array}$ & $\begin{array}{c}-20.36 \\
(-20.49 \text { to }-20.24)\end{array}$ & 36166 & 0.256 \\
\hline 1 & 0.6 & Cost Saving & $\begin{array}{c}2.218 \\
(2.205 \text { to } 2.231)\end{array}$ & $\begin{array}{c}-0.58 \\
(-0.63 \text { to }-0.54)\end{array}$ & $\begin{array}{c}23.42 \\
\text { (23.3 to } 23.54)\end{array}$ & $\begin{array}{c}-25.65 \\
(-25.79 \text { to }-25.51)\end{array}$ & -42907 & 0.789 \\
\hline 1 & 0.8 & Cost Saving & $\begin{array}{c}3.086 \\
(3.071 \text { to } 3.102)\end{array}$ & $\begin{array}{c}-1.15 \\
(-1.21 \text { to }-1.1)\end{array}$ & $\begin{array}{c}31.36 \\
\text { (31.2 to } 31.52)\end{array}$ & $\begin{array}{c}-35.77 \\
(-35.91 \text { to }-35.62)\end{array}$ & -84846 & 0.914 \\
\hline 1 & 1 & Cost Saving & $\begin{array}{c}3.751 \\
(3.734 \text { to } 3.768)\end{array}$ & $\begin{array}{c}-1.98 \\
(-2.04 \text { to }-1.92)\end{array}$ & $\begin{array}{c}35.93 \\
(35.75 \text { to } 36.11)\end{array}$ & $\begin{array}{c}-43.5 \\
(-43.65 \text { to }-43.34)\end{array}$ & -145542 & 0.984 \\
\hline
\end{tabular}


Webappendix Table S12: Simulated health outcomes for Females between 40-69yr across different coverage and adherence scenarios

\begin{tabular}{|c|c|c|c|c|c|c|}
\hline Coverage & Adherence & $\begin{array}{c}\text { Incremental Cost (percent, } \\
95 \% \text { UI) }\end{array}$ & $\begin{array}{l}\text { DALYs Averted } \\
\text { (percent, 95\% UI) }\end{array}$ & $\begin{array}{l}\text { CVD Events Averted } \\
\text { (percent, 95\% UI) }\end{array}$ & $\begin{array}{l}\text { CVD Deaths Averted } \\
\text { (percent, } 95 \% \text { UI) }\end{array}$ & $\begin{array}{c}\text { ICER } \\
\text { (\$/DALY averted) }\end{array}$ \\
\hline 0.4 & 0.4 & $\begin{array}{c}0.87 \\
\text { (0.84 to } 0.9)\end{array}$ & $\begin{array}{c}0.441 \\
(0.431 \text { to } 0.451)\end{array}$ & $\begin{array}{c}1.114 \\
(1.096 \text { to } 1.131)\end{array}$ & $\begin{array}{c}1.166 \\
(1.135 \text { to } 1.198)\end{array}$ & 346.95 \\
\hline 0.4 & 0.6 & $\begin{array}{c}0.33 \\
(0.3 \text { to } 0.36)\end{array}$ & $\begin{array}{c}0.677 \\
(0.666 \text { to } 0.688)\end{array}$ & $\begin{array}{c}1.783 \\
(1.762 \text { to } 1.803)\end{array}$ & $\begin{array}{c}1.862 \\
\text { (1.827 to } 1.897)\end{array}$ & 86.48 \\
\hline 0.4 & 0.8 & $\begin{array}{c}0.05 \\
(0.01 \text { to } 0.08)\end{array}$ & $\begin{array}{c}1.131 \\
(1.118 \text { to } 1.145)\end{array}$ & $\begin{array}{c}3.031 \\
\text { (3.006 to } 3.056)\end{array}$ & $\begin{array}{c}3.147 \\
\text { (3.107 to } 3.186)\end{array}$ & 7.19 \\
\hline 0.4 & 1 & $\begin{array}{c}-0.35 \\
(-0.39 \text { to }-0.31)\end{array}$ & $\begin{array}{c}1.476 \\
(1.462 \text { to } 1.491)\end{array}$ & $\begin{array}{c}3.979 \\
\text { (3.952 to } 4.007)\end{array}$ & $\begin{array}{c}4.11 \\
\text { (4.066 to } 4.154)\end{array}$ & Cost Saving \\
\hline 0.6 & 0.4 & $\begin{array}{c}0.77 \\
(0.73 \text { to } 0.82) \\
\end{array}$ & $\begin{array}{c}1.053 \\
\text { (1.037 to } 1.068)\end{array}$ & $\begin{array}{c}2.775 \\
\text { (2.745 to } 2.804)\end{array}$ & $\begin{array}{c}2.894 \\
\text { (2.846 to } 2.943)\end{array}$ & 128.72 \\
\hline 0.6 & 0.6 & $\begin{array}{c}-0.02 \\
(-0.07 \text { to } 0.03)\end{array}$ & $\begin{array}{c}1.409 \\
(1.392 \text { to } 1.426)\end{array}$ & $\begin{array}{c}3.778 \\
\text { (3.747 to } 3.809)\end{array}$ & $\begin{array}{c}3.917 \\
\text { (3.865 to } 3.968)\end{array}$ & Cost Saving \\
\hline 0.6 & 0.8 & $\begin{array}{c}-0.43 \\
(-0.48 \text { to }-0.37)\end{array}$ & $\begin{array}{c}2.088 \\
\text { (2.069 to } 2.106)\end{array}$ & $\begin{array}{c}5.626 \\
\text { (5.593 to } 5.659)\end{array}$ & $\begin{array}{c}5.84 \\
\text { (5.785 to } 5.895)\end{array}$ & Cost Saving \\
\hline 0.6 & 1 & $\begin{array}{c}-1.04 \\
(-1.1 \text { to }-0.98)\end{array}$ & $\begin{array}{c}2.607 \\
\text { (2.587 to } 2.628)\end{array}$ & $\begin{array}{c}7.055 \\
(7.02 \text { to } 7.09)\end{array}$ & $\begin{array}{c}7.323 \\
(7.265 \text { to } 7.381)\end{array}$ & Cost Saving \\
\hline 0.7 & 0.7 & $\begin{array}{c}-0.46 \\
(-0.51 \text { to }-0.4)\end{array}$ & $\begin{array}{c}2.169 \\
\text { (2.15 to } 2.188)\end{array}$ & $\begin{array}{c}5.852 \\
\text { (5.817 to } 5.887)\end{array}$ & $\begin{array}{c}6.071 \\
(6.013 \text { to } 6.129)\end{array}$ & Cost Saving \\
\hline 0.8 & 0.4 & $\begin{array}{c}0.64 \\
(0.59 \text { to } 0.7)\end{array}$ & $\begin{array}{c}1.676 \\
(1.658 \text { to } 1.694)\end{array}$ & $\begin{array}{c}4.46 \\
\text { (4.428 to } 4.493)\end{array}$ & $\begin{array}{c}4.639 \\
\text { (4.584 to } 4.694)\end{array}$ & 67.34 \\
\hline 0.8 & 0.6 & $\begin{array}{c}-0.39 \\
(-0.45 \text { to }-0.33)\end{array}$ & $\begin{array}{c}2.148 \\
\text { (2.129 to } 2.167)\end{array}$ & $\begin{array}{c}5.778 \\
\text { (5.744 to } 5.812)\end{array}$ & $\begin{array}{c}5.99 \\
\text { (5.933 to } 6.047)\end{array}$ & Cost Saving \\
\hline 0.8 & 0.8 & $\begin{array}{c}-0.96 \\
(-1.02 \text { to }-0.89)\end{array}$ & $\begin{array}{c}3.051 \\
\text { (3.029 to } 3.072)\end{array}$ & $\begin{array}{c}8.25 \\
\text { (8.214 to } 8.287)\end{array}$ & $\begin{array}{c}8.565 \\
(8.504 \text { to } 8.626)\end{array}$ & Cost Saving \\
\hline 0.8 & 1 & $\begin{array}{c}-1.76 \\
(-1.83 \text { to }-1.69)\end{array}$ & $\begin{array}{c}3.741 \\
(3.718 \text { to } 3.764)\end{array}$ & $\begin{array}{c}10.158 \\
(10.121 \text { to } 10.196)\end{array}$ & $\begin{array}{c}10.531 \\
\text { (10.468 to } 10.595)\end{array}$ & Cost Saving \\
\hline 1 & 0.4 & $\begin{array}{c}0.54 \\
(0.48 \text { to } 0.61) \\
\end{array}$ & $\begin{array}{c}2.285 \\
\text { (2.265 to } 2.305) \\
\end{array}$ & $\begin{array}{c}6.133 \\
\text { (6.099 to } 6.168)\end{array}$ & $\begin{array}{c}6.36 \\
\text { (6.301 to } 6.42)\end{array}$ & 41.82 \\
\hline 1 & 0.6 & $\begin{array}{c}-0.76 \\
(-0.82 \text { to }-0.69)\end{array}$ & $\begin{array}{c}2.874 \\
(2.852 \text { to } 2.895)\end{array}$ & $\begin{array}{c}7.786 \\
\text { (7.748 to } 7.824)\end{array}$ & $\begin{array}{c}8.057 \\
\text { (7.994 to } 8.12)\end{array}$ & Cost Saving \\
\hline 1 & 0.8 & $\begin{array}{c}-1.46 \\
(-1.53 \text { to }-1.38)\end{array}$ & $\begin{array}{c}4.005 \\
\text { (3.981 to } 4.029)\end{array}$ & $\begin{array}{c}10.869 \\
\text { (10.83 to } 10.908)\end{array}$ & $\begin{array}{c}11.257 \\
\text { (11.191 to } 11.323)\end{array}$ & Cost Saving \\
\hline 1 & 1 & $\begin{array}{c}-2.46 \\
(-2.54 \text { to }-2.38)\end{array}$ & $\begin{array}{c}4.875 \\
\text { (4.849 to } 4.901)\end{array}$ & $\begin{array}{c}13.247 \\
(13.207 \text { to } 13.286)\end{array}$ & $\begin{array}{c}13.743 \\
(13.674 \text { to } 13.811)\end{array}$ & Cost Saving \\
\hline
\end{tabular}


Webappendix Table S13: Simulated health outcomes for Males between 40-69yr across different coverage and adherence scenarios

\begin{tabular}{|c|c|c|c|c|c|c|}
\hline Coverage & Adherence & $\begin{array}{l}\text { Incremental Cost } \\
\text { (percent, 95\% UI) }\end{array}$ & $\begin{array}{l}\text { DALYs Averted } \\
\text { (percent, 95\% UI) }\end{array}$ & $\begin{array}{l}\text { CVD Events Averted } \\
\text { (percent, 95\% UI) }\end{array}$ & $\begin{array}{l}\text { CVD Deaths Averted } \\
\text { (percent, } 95 \% \text { UI) }\end{array}$ & $\begin{array}{c}\text { ICER } \\
\text { (\$/DALY averted) }\end{array}$ \\
\hline 0.4 & 0.4 & $\begin{array}{c}0.59 \\
(0.57 \text { to } 0.61)\end{array}$ & $\begin{array}{c}0.276 \\
(0.27 \text { to } 0.281)\end{array}$ & $\begin{array}{c}0.705 \\
(0.695 \text { to } 0.715)\end{array}$ & $\begin{array}{c}0.705 \\
(0.688 \text { to } 0.723)\end{array}$ & 354.59 \\
\hline 0.4 & 0.6 & $\begin{array}{c}0.23 \\
(0.21 \text { to } 0.25)\end{array}$ & $\begin{array}{c}0.413 \\
(0.406 \text { to } 0.42)\end{array}$ & $\begin{array}{c}1.138 \\
\text { (1.126 to } 1.151)\end{array}$ & $\begin{array}{c}1.12 \\
\text { (1.098 to } 1.142)\end{array}$ & 91.58 \\
\hline 0.4 & 0.8 & $\begin{array}{c}0.05 \\
(0.02 \text { to } 0.07)\end{array}$ & $\begin{array}{c}0.681 \\
(0.672 \text { to } 0.69)\end{array}$ & $\begin{array}{c}1.942 \\
\text { (1.926 to } 1.958)\end{array}$ & $\begin{array}{c}1.919 \\
(1.892 \text { to } 1.946)\end{array}$ & 10.97 \\
\hline 0.4 & 1 & $\begin{array}{c}-0.22 \\
(-0.24 \text { to }-0.19)\end{array}$ & $\begin{array}{c}0.882 \\
(0.872 \text { to } 0.893)\end{array}$ & $\begin{array}{c}2.553 \\
\text { (2.535 to } 2.572)\end{array}$ & $\begin{array}{c}2.526 \\
\text { (2.495 to } 2.556)\end{array}$ & Cost Saving \\
\hline 0.6 & 0.4 & $\begin{array}{c}0.61 \\
(0.57 \text { to } 0.64) \\
\end{array}$ & $\begin{array}{c}0.641 \\
(0.63 \text { to } 0.653)\end{array}$ & $\begin{array}{c}1.747 \\
\text { (1.723 to } 1.77)\end{array}$ & $\begin{array}{c}1.767 \\
\text { (1.731 to } 1.803)\end{array}$ & 156.78 \\
\hline 0.6 & 0.6 & $\begin{array}{c}0.07 \\
(0.03 \text { to } 0.1)\end{array}$ & $\begin{array}{c}0.845 \\
\text { (0.833 to } 0.858)\end{array}$ & $\begin{array}{c}2.394 \\
\text { (2.369 to } 2.419)\end{array}$ & $\begin{array}{c}2.388 \\
\text { (2.35 to } 2.426)\end{array}$ & 13.06 \\
\hline 0.6 & 0.8 & $\begin{array}{c}-0.21 \\
(-0.26 \text { to }-0.17)\end{array}$ & $\begin{array}{c}1.25 \\
\text { (1.236 to } 1.264)\end{array}$ & $\begin{array}{c}3.611 \\
\text { (3.584 to } 3.638)\end{array}$ & $\begin{array}{c}3.605 \\
\text { (3.563 to } 3.648)\end{array}$ & Cost Saving \\
\hline 0.6 & 1 & $\begin{array}{c}-0.63 \\
(-0.68 \text { to }-0.59)\end{array}$ & $\begin{array}{c}1.557 \\
\text { (1.541 to } 1.572)\end{array}$ & $\begin{array}{c}4.542 \\
\text { (4.513 to } 4.571)\end{array}$ & $\begin{array}{c}4.513 \\
\text { (4.47 to } 4.557)\end{array}$ & Cost Saving \\
\hline 0.7 & 0.7 & $\begin{array}{c}-0.29 \\
(-0.33 \text { to }-0.24)\end{array}$ & $\begin{array}{c}1.303 \\
(1.289 \text { to } 1.316)\end{array}$ & $\begin{array}{c}3.78 \\
\text { (3.752 to } 3.808)\end{array}$ & $\begin{array}{c}3.756 \\
\text { (3.714 to } 3.799)\end{array}$ & Cost Saving \\
\hline 0.8 & 0.4 & $\begin{array}{c}0.48 \\
(0.43 \text { to } 0.52)\end{array}$ & $\begin{array}{c}1.012 \\
(0.999 \text { to } 1.025)\end{array}$ & $\begin{array}{c}2.875 \\
\text { (2.849 to } 2.901)\end{array}$ & $\begin{array}{c}2.882 \\
\text { (2.842 to } 2.922)\end{array}$ & 78.13 \\
\hline 0.8 & 0.6 & $\begin{array}{c}-0.24 \\
(-0.29 \text { to }-0.2)\end{array}$ & $\begin{array}{c}1.284 \\
(1.27 \text { to } 1.299)\end{array}$ & $\begin{array}{c}3.739 \\
(3.712 \text { to } 3.767)\end{array}$ & $\begin{array}{c}3.713 \\
(3.671 \text { to } 3.755)\end{array}$ & Cost Saving \\
\hline 0.8 & 0.8 & $\begin{array}{c}-0.6 \\
(-0.65 \text { to }-0.55)\end{array}$ & $\begin{array}{c}1.812 \\
\text { (1.797 to } 1.827)\end{array}$ & $\begin{array}{c}5.335 \\
(5.305 \text { to } 5.364)\end{array}$ & $\begin{array}{c}5.301 \\
(5.255 \text { to } 5.346)\end{array}$ & Cost Saving \\
\hline 0.8 & 1 & $\begin{array}{c}-1.13 \\
(-1.19 \text { to }-1.08)\end{array}$ & $\begin{array}{c}2.214 \\
\text { (2.197 to } 2.23)\end{array}$ & $\begin{array}{c}6.557 \\
\text { (6.527 to } 6.588)\end{array}$ & $\begin{array}{c}6.5 \\
\text { (6.453 to } 6.548)\end{array}$ & Cost Saving \\
\hline 1 & 0.4 & $\begin{array}{c}0.45 \\
(0.4 \text { to } 0.5)\end{array}$ & $\begin{array}{c}1.372 \\
(1.359 \text { to } 1.386)\end{array}$ & $\begin{array}{c}3.942 \\
(3.916 \text { to } 3.969)\end{array}$ & $\begin{array}{c}3.954 \\
(3.912 \text { to } 3.996)\end{array}$ & 54.35 \\
\hline 1 & 0.6 & $\begin{array}{c}-0.44 \\
(-0.49 \text { to }-0.39)\end{array}$ & $\begin{array}{c}1.711 \\
\text { (1.696 to } 1.726)\end{array}$ & $\begin{array}{c}5.005 \\
\text { (4.978 to } 5.033)\end{array}$ & $\begin{array}{c}4.997 \\
\text { (4.952 to } 5.042)\end{array}$ & Cost Saving \\
\hline 1 & 0.8 & $\begin{array}{c}-0.9 \\
(-0.96 \text { to }-0.85)\end{array}$ & $\begin{array}{c}2.377 \\
\text { (2.36 to } 2.394)\end{array}$ & $\begin{array}{c}7.023 \\
\text { (6.993 to } 7.053)\end{array}$ & $\begin{array}{c}6.986 \\
\text { (6.938 to } 7.034)\end{array}$ & Cost Saving \\
\hline 1 & 1 & $\begin{array}{c}-1.58 \\
(-1.64 \text { to }-1.52)\end{array}$ & $\begin{array}{c}2.882 \\
\text { (2.864 to } 2.901)\end{array}$ & $\begin{array}{c}8.566 \\
\text { (8.534 to } 8.598)\end{array}$ & $\begin{array}{c}8.499 \\
\text { (8.45 to } 8.548)\end{array}$ & Cost Saving \\
\hline
\end{tabular}




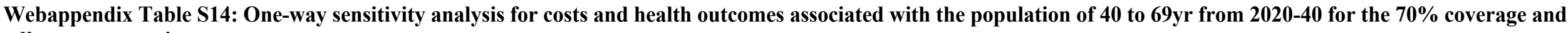
adherence scenario

\begin{tabular}{|c|c|c|c|c|c|c|}
\hline \multirow{2}{*}{ Sensitivity Scenario } & \multirow{2}{*}{$\begin{array}{c}\text { ICER } \\
\text { (\$/DALY averted) }\end{array}$} & \multirow{2}{*}{$\begin{array}{l}\text { DALYs Averted } \\
\text { (percent, 95\% UI) }\end{array}$} & \multicolumn{2}{|c|}{$\begin{array}{l}\text { Per-capita incremental } \\
\text { costs over } 20 \text { years }\end{array}$} & \multirow{2}{*}{$\begin{array}{l}\text { Annual net expenditure for } \\
\text { 40-69yr population } \\
\text { (in '000 US \$) }\end{array}$} & \multirow{2}{*}{$\begin{array}{l}\text { Probability of } \\
\text { Cost Saving }\end{array}$} \\
\hline & & & $\begin{array}{c}\text { Antihypertensive Program } \\
(\$, 95 \% \text { UI })\end{array}$ & $\begin{array}{l}\text { CVD Treatment } \\
(\$, 95 \% \text { UI })\end{array}$ & & \\
\hline $\begin{array}{l}\text { Results using main input } \\
\text { assumptions }\end{array}$ & Cost Saving & $\begin{array}{c}1.68 \\
\text { (1.668 to } 1.692)\end{array}$ & $\begin{array}{c}-0.36 \\
(-0.4 \text { to }-0.33)\end{array}$ & $\begin{array}{c}18.04 \\
(17.95 \text { to } 18.13)\end{array}$ & $\begin{array}{c}-19.43 \\
(-19.55 \text { to }-19.3)\end{array}$ & 0.721 \\
\hline $\begin{array}{l}\text { 2X Antihypertensive } \\
\text { Medication Cost }\end{array}$ & 106.92 & $\begin{array}{c}1.68 \\
\text { (1.668 to } 1.692)\end{array}$ & $\begin{array}{c}1.05 \\
\text { (1.01 to } 1.09)\end{array}$ & $\begin{array}{c}23.44 \\
\text { (23.35 to } 23.54)\end{array}$ & $\begin{array}{c}-19.41 \\
(-19.54 \text { to }-19.29)\end{array}$ & 0.039 \\
\hline 4X Programmatic Cost & 121.64 & $\begin{array}{c}1.68 \\
\text { (1.668 to } 1.692)\end{array}$ & $\begin{array}{c}1.2 \\
\text { (1.16 to } 1.24)\end{array}$ & $\begin{array}{c}24.01 \\
\text { (23.92 to } 24.11)\end{array}$ & $\begin{array}{c}-19.43 \\
(-19.55 \text { to }-19.3)\end{array}$ & 0.025 \\
\hline $\begin{array}{l}\text { 20\% Reduction in } \\
\text { Baseline CVD Risk }\end{array}$ & 58.00 & $\begin{array}{c}1.49 \\
(1.479 \text { to } 1.502)\end{array}$ & $\begin{array}{c}0.58 \\
\text { (0.54 to } 0.62)\end{array}$ & $\begin{array}{c}18.37 \\
\text { (18.28 to } 18.46)\end{array}$ & $\begin{array}{c}-16.55 \\
(-16.66 \text { to }-16.43)\end{array}$ & 0.201 \\
\hline $\begin{array}{l}\text { NPCDCS Medication } \\
\text { Guideline }\end{array}$ & Cost Saving & $\begin{array}{c}1.175 \\
(1.166 \text { to } 1.185)\end{array}$ & $\begin{array}{c}-0.84 \\
(-0.87 \text { to }-0.81)\end{array}$ & $\begin{array}{c}10.76 \\
\text { (10.72 to } 10.8)\end{array}$ & $\begin{array}{c}-13.97 \\
(-14.08 \text { to }-13.86)\end{array}$ & 0.967 \\
\hline Only Private Sector & 444.38 & $\begin{array}{c}1.623 \\
\text { (1.611 to } 1.635)\end{array}$ & $\begin{array}{c}2.8 \\
(2.77 \text { to } 2.84)\end{array}$ & $\begin{array}{c}44.33 \\
(44.21 \text { to } 44.45)\end{array}$ & $\begin{array}{c}-28.14 \\
(-28.33 \text { to }-27.95)\end{array}$ & 0 \\
\hline $\begin{array}{l}\text { Public-Private Sector } \\
\text { Mix }\end{array}$ & 345.46 & $\begin{array}{c}1.634 \\
\text { (1.622 to } 1.646)\end{array}$ & $\begin{array}{c}2.35 \\
\text { (2.32 to } 2.39)\end{array}$ & $\begin{array}{c}39.07 \\
(38.96 \text { to } 39.18)\end{array}$ & $\begin{array}{c}-26.4 \\
(-26.58 \text { to }-26.22)\end{array}$ & 0 \\
\hline 10 year Time Horizon & 134.38 & $\begin{array}{c}1.885 \\
\text { (1.865 to } 1.905)\end{array}$ & $\begin{array}{c}0.8 \\
(0.75 \text { to } 0.85)\end{array}$ & $\begin{array}{c}11.54 \\
(11.47 \text { to } 11.6)\end{array}$ & $\begin{array}{c}-10.09 \\
(-10.18 \text { to }-10.01)\end{array}$ & 0.192 \\
\hline 40 year Time Horizon & Cost Saving & $\begin{array}{c}1.073 \\
\text { (1.067 to } 1.079)\end{array}$ & $\begin{array}{c}-0.4 \\
(-0.43 \text { to }-0.37)\end{array}$ & $\begin{array}{c}23.3 \\
\text { (23.19 to } 23.41)\end{array}$ & $\begin{array}{c}-25.56 \\
(-25.71 \text { to }-25.41)\end{array}$ & 0.786 \\
\hline
\end{tabular}


Webappendix Table S15: Simulated health outcomes for Females between 40-69yr across one-way sensitivity analysis

\begin{tabular}{|c|c|c|c|c|c|}
\hline Sensitivity Scenario & $\begin{array}{l}\text { Incremental Cost } \\
\text { (percent, } 95 \% \text { UI) }\end{array}$ & $\begin{array}{l}\text { DALYs Averted } \\
\text { (percent, 95\% UI) }\end{array}$ & $\begin{array}{l}\text { CVD Events Averted } \\
\text { (percent, } 95 \% \text { UI) }\end{array}$ & $\begin{array}{l}\text { CVD Deaths Averted } \\
\text { (percent, } 95 \% \text { UI) }\end{array}$ & $\begin{array}{c}\text { ICER } \\
\text { (\$/DALY averted) }\end{array}$ \\
\hline $\begin{array}{l}\text { Results using main input } \\
\text { assumptions }\end{array}$ & $\begin{array}{c}-0.46 \\
(-0.51 \text { to }-0.4)\end{array}$ & $\begin{array}{c}2.169 \\
\text { (2.15 to } 2.188)\end{array}$ & $\begin{array}{c}5.852 \\
\text { (5.817 to } 5.887)\end{array}$ & $\begin{array}{c}6.071 \\
(6.013 \text { to } 6.129)\end{array}$ & Cost Saving \\
\hline $\begin{array}{l}\text { 2X Antihypertensive } \\
\text { Medication Cost }\end{array}$ & $\begin{array}{c}1.47 \\
(1.42 \text { to } 1.53)\end{array}$ & $\begin{array}{c}2.169 \\
\text { (2.15 to } 2.188)\end{array}$ & $\begin{array}{c}5.852 \\
(5.817 \text { to } 5.887)\end{array}$ & $\begin{array}{c}6.071 \\
(6.013 \text { to } 6.129)\end{array}$ & 120.18 \\
\hline 4X Programmatic Cost & $\begin{array}{c}1.24 \\
\text { (1.19 to } 1.3)\end{array}$ & $\begin{array}{c}2.169 \\
\text { (2.15 to } 2.188)\end{array}$ & $\begin{array}{c}5.852 \\
\text { (5.817 to } 5.887)\end{array}$ & $\begin{array}{c}6.071 \\
(6.013 \text { to } 6.129)\end{array}$ & 100.74 \\
\hline $\begin{array}{l}20 \% \text { Reduction in Baseline } \\
\text { CVD Risk }\end{array}$ & $\begin{array}{c}0.73 \\
(0.67 \text { to } 0.8)\end{array}$ & $\begin{array}{c}1.918 \\
\text { (1.899 to } 1.937)\end{array}$ & $\begin{array}{c}6.174 \\
(6.135 \text { to } 6.214)\end{array}$ & $\begin{array}{c}6.21 \\
(6.146 \text { to } 6.274)\end{array}$ & 58.72 \\
\hline NPCDCS Medication Guideline & $\begin{array}{c}-1.24 \\
(-1.29 \text { to }-1.2)\end{array}$ & $\begin{array}{c}1.559 \\
(1.542 \text { to } 1.575)\end{array}$ & $\begin{array}{c}4.411 \\
(4.38 \text { to } 4.442)\end{array}$ & $\begin{array}{c}4.577 \\
\text { (4.525 to } 4.628)\end{array}$ & Cost Saving \\
\hline Only Private Sector & $\begin{array}{c}4.24 \\
(4.18 \text { to } 4.29)\end{array}$ & $\begin{array}{c}2.094 \\
\text { (2.075 to } 2.113)\end{array}$ & $\begin{array}{c}5.559 \\
\text { (5.525 to } 5.593)\end{array}$ & $\begin{array}{c}5.752 \\
\text { (5.695 to } 5.809)\end{array}$ & 540.39 \\
\hline Public-Private Sector Mix & $\begin{array}{c}3.57 \\
\text { (3.52 to } 3.63)\end{array}$ & $\begin{array}{c}2.109 \\
\text { (2.09 to } 2.128)\end{array}$ & $\begin{array}{c}5.617 \\
\text { (5.584 to } 5.651)\end{array}$ & $\begin{array}{c}5.816 \\
\text { (5.758 to } 5.873)\end{array}$ & 421.64 \\
\hline 10 year Time Horizon & $\begin{array}{c}1.07 \\
(0.98 \text { to } 1.15)\end{array}$ & $\begin{array}{c}2.452 \\
\text { (2.419 to } 2.485)\end{array}$ & $\begin{array}{c}6.871 \\
(6.817 \text { to } 6.925)\end{array}$ & $\begin{array}{c}6.967 \\
\text { (6.858 to } 7.076)\end{array}$ & 142.15 \\
\hline 40 year Time Horizon & $\begin{array}{c}-0.42 \\
(-0.46 \text { to }-0.37)\end{array}$ & $\begin{array}{c}1.331 \\
(1.322 \text { to } 1.34)\end{array}$ & $\begin{array}{c}4.278 \\
\text { (4.254 to } 4.302)\end{array}$ & $\begin{array}{c}4.454 \\
\text { (4.422 to } 4.486)\end{array}$ & Cost Saving \\
\hline
\end{tabular}


Webappendix Table S16: Simulated health outcomes for Males between 40-69yr across one-way sensitivity analysis

\begin{tabular}{|c|c|c|c|c|c|}
\hline Sensitivity Scenario & $\begin{array}{l}\text { Incremental Cost } \\
\text { (percent, } 95 \% \text { UI) }\end{array}$ & $\begin{array}{l}\text { DALYs Averted } \\
\text { (percent, 95\% UI) }\end{array}$ & $\begin{array}{l}\text { CVD Events Averted } \\
\text { (percent, } 95 \% \text { UI) }\end{array}$ & $\begin{array}{l}\text { CVD Deaths Averted } \\
\text { (percent, } 95 \% \text { UI) }\end{array}$ & $\begin{array}{c}\text { ICER } \\
\text { (\$/DALY averted) }\end{array}$ \\
\hline $\begin{array}{l}\text { Results using main input } \\
\text { assumptions }\end{array}$ & $\begin{array}{c}-0.29 \\
(-0.33 \text { to }-0.24)\end{array}$ & $\begin{array}{c}1.303 \\
(1.289 \text { to } 1.316)\end{array}$ & $\begin{array}{c}3.78 \\
(3.752 \text { to } 3.808)\end{array}$ & $\begin{array}{c}3.756 \\
(3.714 \text { to } 3.799)\end{array}$ & Cost Saving \\
\hline $\begin{array}{l}\text { 2X Antihypertensive } \\
\text { Medication Cost }\end{array}$ & $\begin{array}{c}0.7 \\
(0.66 \text { to } 0.75)\end{array}$ & $\begin{array}{c}1.303 \\
(1.289 \text { to } 1.316)\end{array}$ & $\begin{array}{c}3.78 \\
\text { (3.752 to } 3.808)\end{array}$ & $\begin{array}{c}3.756 \\
\text { (3.714 to } 3.799)\end{array}$ & 89.89 \\
\hline 4X Programmatic Cost & $\begin{array}{c}1.17 \\
(1.12 \text { to } 1.21)\end{array}$ & $\begin{array}{c}1.303 \\
(1.289 \text { to } 1.316)\end{array}$ & $\begin{array}{c}3.78 \\
(3.752 \text { to } 3.808)\end{array}$ & $\begin{array}{c}3.756 \\
(3.714 \text { to } 3.799)\end{array}$ & 148.50 \\
\hline $\begin{array}{l}20 \% \text { Reduction in Baseline } \\
\text { CVD Risk }\end{array}$ & $\begin{array}{c}0.46 \\
(0.41 \text { to } 0.51)\end{array}$ & $\begin{array}{c}1.161 \\
(1.148 \text { to } 1.174)\end{array}$ & $\begin{array}{c}4.067 \\
\text { (4.037 to } 4.097)\end{array}$ & $\begin{array}{c}3.915 \\
\text { (3.868 to } 3.963)\end{array}$ & 57.08 \\
\hline NPCDCS Medication Guideline & $\begin{array}{c}-0.51 \\
(-0.54 \text { to }-0.48)\end{array}$ & $\begin{array}{c}0.88 \\
(0.868 \text { to } 0.891)\end{array}$ & $\begin{array}{c}2.87 \\
\text { (2.845 to } 2.895)\end{array}$ & $\begin{array}{c}2.804 \\
\text { (2.766 to } 2.841)\end{array}$ & Cost Saving \\
\hline Only Private Sector & $\begin{array}{c}1.62 \\
(1.58 \text { to } 1.66)\end{array}$ & $\begin{array}{c}1.259 \\
(1.245 \text { to } 1.272)\end{array}$ & $\begin{array}{c}3.595 \\
\text { (3.568 to } 3.622)\end{array}$ & $\begin{array}{c}3.553 \\
\text { (3.511 to } 3.594)\end{array}$ & 321.09 \\
\hline Public-Private Sector Mix & $\begin{array}{c}1.35 \\
(1.31 \text { to } 1.39)\end{array}$ & $\begin{array}{c}1.267 \\
(1.254 \text { to } 1.281)\end{array}$ & $\begin{array}{c}3.632 \\
(3.605 \text { to } 3.659)\end{array}$ & $\begin{array}{c}3.594 \\
(3.552 \text { to } 3.635)\end{array}$ & 247.61 \\
\hline 10 year Time Horizon & $\begin{array}{c}0.6 \\
(0.54 \text { to } 0.66)\end{array}$ & $\begin{array}{c}1.472 \\
(1.45 \text { to } 1.494)\end{array}$ & $\begin{array}{c}4.319 \\
(4.281 \text { to } 4.357)\end{array}$ & $\begin{array}{c}4.291 \\
(4.215 \text { to } 4.367)\end{array}$ & 124.96 \\
\hline 40 year Time Horizon & $\begin{array}{c}-0.38 \\
(-0.42 \text { to }-0.35)\end{array}$ & $\begin{array}{c}0.854 \\
(0.847 \text { to } 0.861)\end{array}$ & $\begin{array}{c}3.319 \\
\text { (3.297 to } 3.34)\end{array}$ & $\begin{array}{c}3.261 \\
\text { (3.234 to } 3.287)\end{array}$ & Cost Saving \\
\hline
\end{tabular}


Webappendix Figure 1: Schematic of Model Structure

\section{Scenario Options}

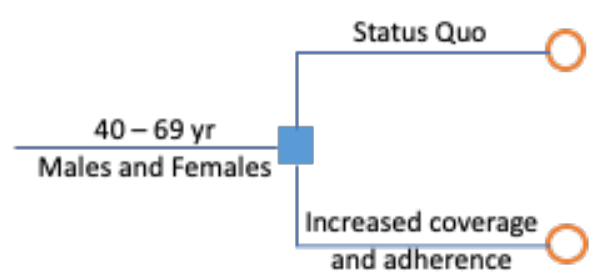

\section{Transition from Well state}

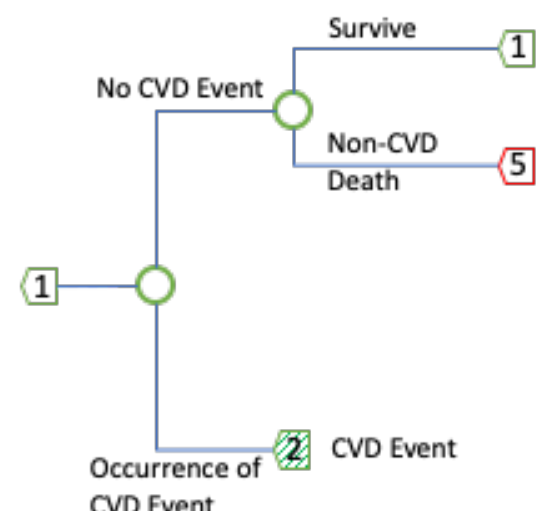

\section{Health States}

\section{considered in the model}

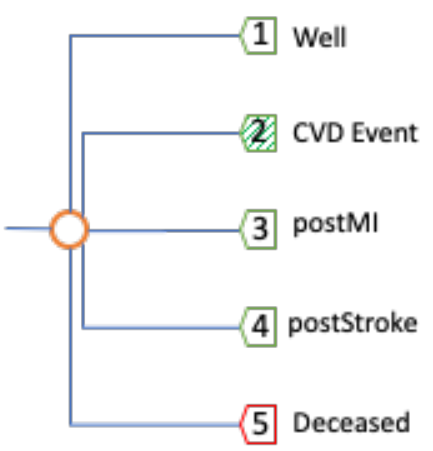

\section{Transition from occurrence of a CVD event}

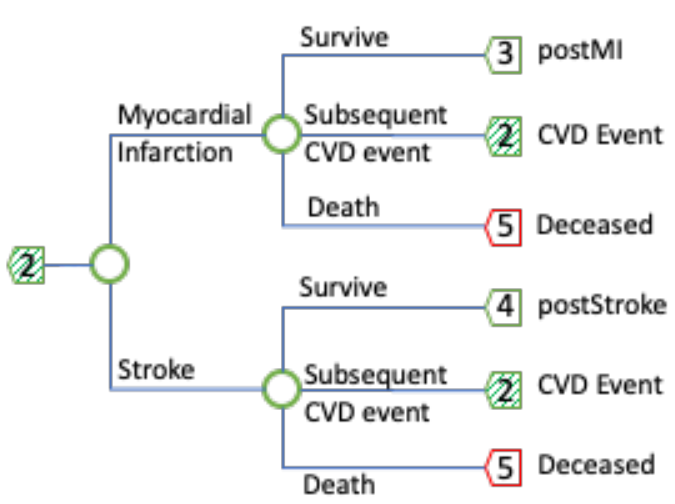

\section{Transition from postMI and postStroke states}

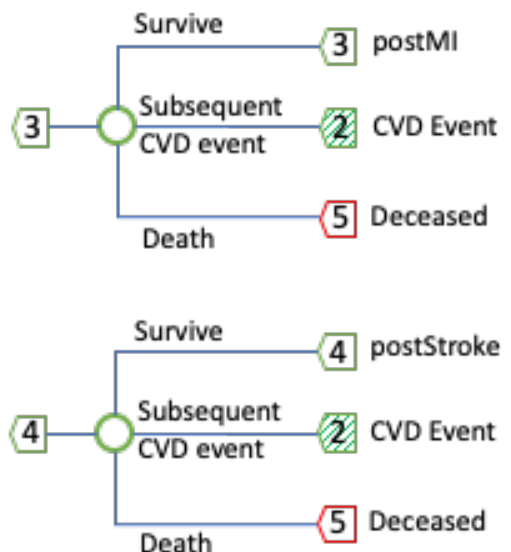

The blue square indicates the choice between various intervention scenarios, and the orange circle indicates the chosen intervention. The health states (indicated by green pentagon) comprises of (1) well (no past CVD event), (2) occurrence of a CVD event, (3) surviving post a myocardial infarction (postMI), (4) surviving post a stroke (postStroke), and (5) deceased state. The blue-colored branches from each heath state lead to another heath state based on the probability of the intermediate event (indicated by the green circle). The (2) CVD Event is a transitionary markov state and comprises of either an occurrence of MI or stroke. 


\section{Webappendix Figure 2: Validation of model output versus GBD 2017 estimates}

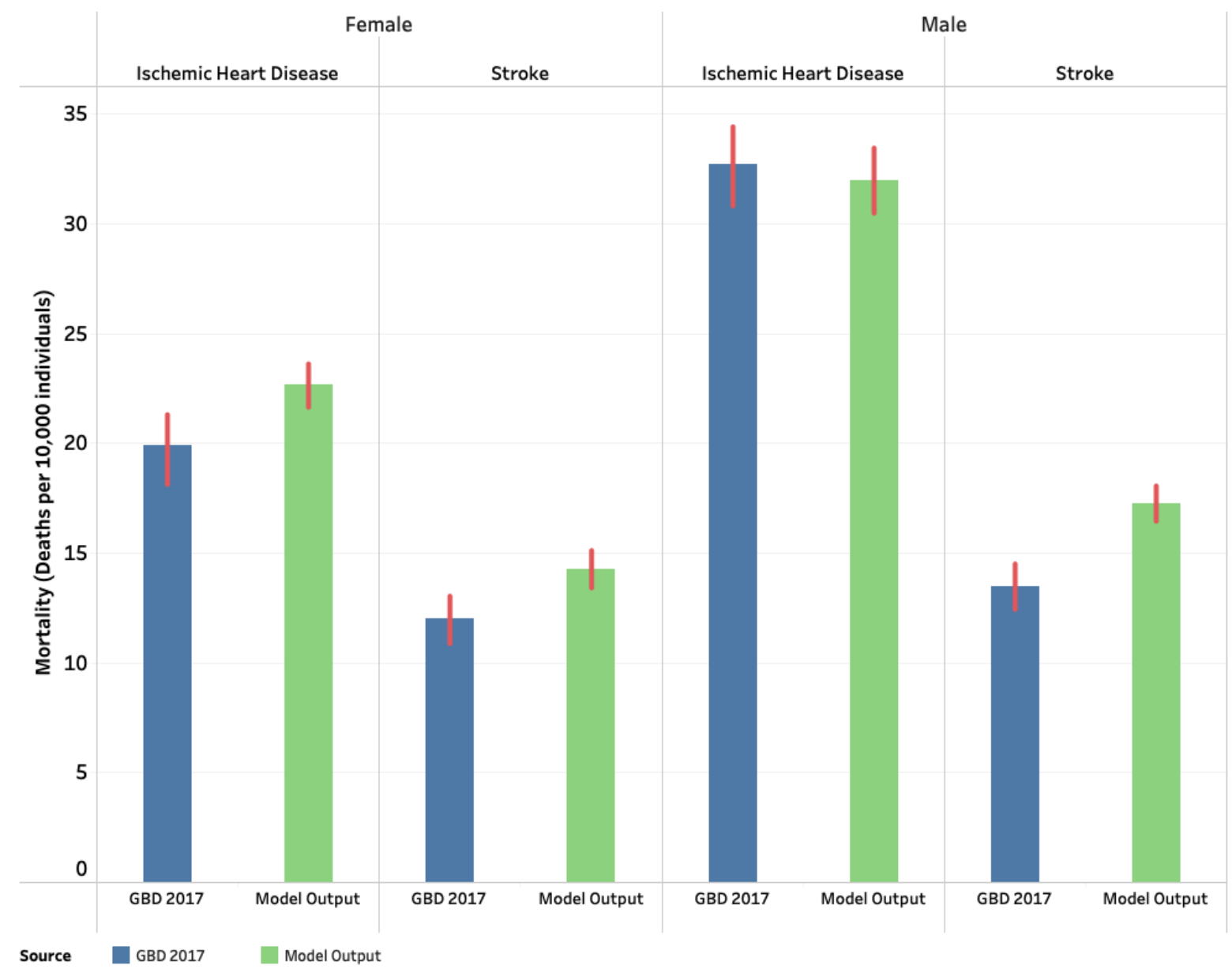

The figure provides the average deaths predicted by the microsimulation model on average over 5 years. We compare our predicted values with the Global Burden of Disease study's estimated deaths for 2017. The blue bar and green bar represent the mean deaths for GBD 2017 and model output respectively. The red line represents the $95 \%$ confidence intervals. The estimates have been disaggregated by sex and cause of death (Ischemic Heart Disease or Stroke). 


\section{Webappendix Table 17: Consolidated Health Economic Evaluation Reporting Standards (CHEERS) checklist [27]}

\begin{tabular}{|c|c|c|c|}
\hline Section/item & $\begin{array}{l}\text { Item } \\
\text { No }\end{array}$ & Recommendation & $\begin{array}{l}\text { Reported on page } \\
\text { No/ line No }\end{array}$ \\
\hline \multicolumn{4}{|l|}{ Title and abstract } \\
\hline Title & 1 & $\begin{array}{l}\text { Identify the study as an economic evaluation or use more specific } \\
\text { terms such as "cost-effectiveness analysis", and describe the } \\
\text { interventions compared. }\end{array}$ & Page\# 1 \\
\hline Abstract & 2 & $\begin{array}{l}\text { Provide a structured summary of objectives, perspective, setting, } \\
\text { methods (including study design and inputs), results (including base } \\
\text { case and uncertainty analyses), and conclusions. }\end{array}$ & Page\# 2 \\
\hline \multicolumn{4}{|l|}{ Introduction } \\
\hline \multirow{2}{*}{$\begin{array}{l}\text { Background and } \\
\text { objectives }\end{array}$} & \multirow[t]{2}{*}{3} & Provide an explicit statement of the broader context for the study. & $10-16$ \\
\hline & & $\begin{array}{l}\text { Present the study question and its relevance for health policy or } \\
\text { practice decisions. }\end{array}$ & $35-40$ \\
\hline \multicolumn{4}{|l|}{ Methods } \\
\hline $\begin{array}{l}\text { Target population } \\
\text { and subgroups }\end{array}$ & 4 & $\begin{array}{l}\text { Describe characteristics of the base case population and subgroups } \\
\text { analysed, including why they were chosen. }\end{array}$ & 44-46, Table 1 \\
\hline Setting and location & 5 & $\begin{array}{l}\text { State relevant aspects of the system(s) in which the decision(s) } \\
\text { need(s) to be made. }\end{array}$ & $46-48$ \\
\hline Study perspective & 6 & $\begin{array}{l}\text { Describe the perspective of the study and relate this to the costs } \\
\text { being evaluated. }\end{array}$ & 49,50 \\
\hline Comparators & 7 & $\begin{array}{l}\text { Describe the interventions or strategies being compared and state } \\
\text { why they were chosen. }\end{array}$ & $97-122$ \\
\hline Time horizon & 8 & $\begin{array}{l}\text { State the time horizon(s) over which costs and consequences are } \\
\text { being evaluated and say why appropriate. }\end{array}$ & 46,47 \\
\hline Discount rate & 9 & $\begin{array}{l}\text { Report the choice of discount rate(s) used for costs and outcomes } \\
\text { and say why appropriate. }\end{array}$ & 94,95 \\
\hline $\begin{array}{l}\text { Choice of health } \\
\text { outcomes }\end{array}$ & 10 & $\begin{array}{l}\text { Describe what outcomes were used as the measure(s) of benefit in } \\
\text { the evaluation and their relevance for the type of analysis } \\
\text { performed. }\end{array}$ & $46-49,136-141$ \\
\hline \multirow[t]{2}{*}{$\begin{array}{l}\text { Measurement of } \\
\text { effectiveness }\end{array}$} & $11 \mathrm{a}$ & $\begin{array}{l}\text { Single study-based estimates: Describe fully the design features of } \\
\text { the single effectiveness study and why the single study was a } \\
\text { sufficient source of clinical effectiveness data. }\end{array}$ & NA \\
\hline & $11 \mathrm{~b}$ & $\begin{array}{l}\text { Synthesis-based estimates: Describe fully the methods used for } \\
\text { identification of included studies and synthesis of clinical } \\
\text { effectiveness data. }\end{array}$ & $\begin{array}{l}\text { 60-71, Table } 1, \\
\text { Webappendix Table } \\
\text { S7 }\end{array}$ \\
\hline $\begin{array}{l}\text { Measurement and } \\
\text { valuation of } \\
\text { preference based } \\
\text { outcomes }\end{array}$ & 12 & $\begin{array}{l}\text { If applicable, describe the population and methods used to elicit } \\
\text { preferences for outcomes. }\end{array}$ & NA \\
\hline \multirow[t]{2}{*}{$\begin{array}{l}\text { Estimating resources } \\
\text { and costs }\end{array}$} & $13 \mathrm{a}$ & $\begin{array}{l}\text { Single study-based economic evaluation: Describe approaches used } \\
\text { to estimate resource use associated with the alternative } \\
\text { interventions. Describe primary or secondary research methods for } \\
\text { valuing each resource item in terms of its unit cost. Describe any } \\
\text { adjustments made to approximate to opportunity costs. }\end{array}$ & NA \\
\hline & $13 b$ & $\begin{array}{l}\text { Model-based economic evaluation: Describe approaches and data } \\
\text { sources used to estimate resource use associated with model health } \\
\text { states. Describe primary or secondary research methods for valuing } \\
\text { each resource item in terms of its unit cost. Describe any } \\
\text { adjustments made to approximate to opportunity costs. }\end{array}$ & $\begin{array}{l}\text { 73-89, Table } 1, \\
\text { Webappendix Table } \\
\text { S8 - S10 }\end{array}$ \\
\hline $\begin{array}{l}\text { Currency, price date, } \\
\text { and conversion }\end{array}$ & 14 & $\begin{array}{l}\text { Report the dates of the estimated resource quantities and unit costs. } \\
\text { Describe methods for adjusting estimated unit costs to the year of } \\
\text { reported costs if necessary. Describe methods for converting costs } \\
\text { into a common currency base and the exchange rate. }\end{array}$ & 88,89 \\
\hline Choice of model & 15 & $\begin{array}{l}\text { Describe and give reasons for the specific type of decision- } \\
\text { analytical model used. Providing a figure to show model structure is } \\
\text { strongly recommended. }\end{array}$ & $\begin{array}{l}\text { 43-58, Figure 1, } \\
\text { Webappendix 1-24, } \\
\text { Webappendix Table } \\
\text { S1 }\end{array}$ \\
\hline Assumptions & 16 & $\begin{array}{l}\text { Describe all structural or other assumptions underpinning the } \\
\text { decision-analytical model. }\end{array}$ & 52-71, Figure 1 \\
\hline Analytical methods & 17 & $\begin{array}{l}\text { Describe all analytical methods supporting the evaluation. This } \\
\text { could include methods for dealing with skewed, missing, or } \\
\text { censored data; extrapolation methods; methods for pooling data; } \\
\text { approaches to validate or make adjustments (such as half cycle }\end{array}$ & $43-71,97-134$ \\
\hline
\end{tabular}


corrections) to a model; and methods for handling population heterogeneity and uncertainty.

\begin{tabular}{|c|c|c|c|}
\hline & & $\begin{array}{l}\text { corrections) to a model; and methods for handling population } \\
\text { heterogeneity and uncertainty. }\end{array}$ & \\
\hline \multicolumn{4}{|l|}{ Results } \\
\hline Study parameters & 18 & $\begin{array}{l}\text { Report the values, ranges, references, and, if used, probability } \\
\text { distributions for all parameters. Report reasons or sources for } \\
\text { distributions used to represent uncertainty where appropriate. } \\
\text { Providing a table to show the input values is strongly recommended. }\end{array}$ & $\begin{array}{l}\text { 43-134, Table 1, } \\
\text { Webappendix Table } \\
\text { S2 - S6 }\end{array}$ \\
\hline $\begin{array}{l}\text { Incremental costs } \\
\text { and outcomes }\end{array}$ & 19 & $\begin{array}{l}\text { For each intervention, report mean values for the main categories of } \\
\text { estimated costs and outcomes of interest, as well as mean } \\
\text { differences between the comparator groups. If applicable, report } \\
\text { incremental cost-effectiveness ratios. }\end{array}$ & $\begin{array}{l}\text { 151-161, Table 2, } \\
\text { Webappendix Table } \\
\text { S11 }\end{array}$ \\
\hline \multirow[t]{2}{*}{$\begin{array}{l}\text { Characterising } \\
\text { uncertainty }\end{array}$} & $20 \mathrm{a}$ & $\begin{array}{l}\text { Single study-based economic evaluation: Describe the effects of } \\
\text { sampling uncertainty for the estimated incremental cost and } \\
\text { incremental effectiveness parameters, together with the impact of } \\
\text { methodological assumptions (such as discount rate, study } \\
\text { perspective). }\end{array}$ & NA \\
\hline & $20 \mathrm{~b}$ & $\begin{array}{l}\text { Model-based economic evaluation: Describe the effects on the } \\
\text { results of uncertainty for all input parameters, and uncertainty } \\
\text { related to the structure of the model and assumptions. }\end{array}$ & $\begin{array}{l}\text { 182-201, Figure 3, } \\
\text { Webappendix Table } \\
\text { S14-S16 }\end{array}$ \\
\hline $\begin{array}{l}\text { Characterising } \\
\text { heterogeneity }\end{array}$ & 21 & $\begin{array}{l}\text { If applicable, report differences in costs, outcomes, or cost- } \\
\text { effectiveness that can be explained by variations between subgroups } \\
\text { of patients with different baseline characteristics or other observed } \\
\text { variability in effects that are not reducible by more information. }\end{array}$ & $\begin{array}{l}\text { 151-174, Table 2, } \\
\text { Figure 2, } \\
\text { Webappendix Table } \\
\text { S12-S13 }\end{array}$ \\
\hline \multicolumn{4}{|l|}{ Discussion } \\
\hline $\begin{array}{l}\text { Study findings, } \\
\text { limitations, } \\
\text { generalisability, and } \\
\text { current knowledge }\end{array}$ & 22 & $\begin{array}{l}\text { Summarise key study findings and describe how they support the } \\
\text { conclusions reached. Discuss limitations and the generalisability of } \\
\text { the findings and how the findings fit with current knowledge. }\end{array}$ & $203-304$ \\
\hline \multicolumn{4}{|l|}{ Other } \\
\hline Source of funding & 23 & $\begin{array}{l}\text { Describe how the study was funded and the role of the funder in the } \\
\text { identification, design, conduct, and reporting of the analysis. } \\
\text { Describe other non-monetary sources of support. }\end{array}$ & 321 \\
\hline Conflicts of interest & 24 & $\begin{array}{l}\text { Describe any potential for conflict of interest of study contributors } \\
\text { in accordance with journal policy. In the absence of a journal } \\
\text { policy, we recommend authors comply with International } \\
\text { Committee of Medical Journal Editors recommendations. }\end{array}$ & $323-331$ \\
\hline
\end{tabular}




\section{References}

[1] Kowal P, Chatterji S, Naidoo N, Biritwum R, Fan W, Lopez Ridaura R, et al. Data Resource Profile: The World Health Organization Study on global AGEing and adult health (SAGE). Int J Epidemiol 2012;41:1639-49. https://doi.org/10.1093/ije/dys210.

[2] WHO. Global Health Observatory data repository. Geneva: World Health Organization; 2018.

[3] Global Burden of Disease Collaborative Network. Global Burden of Disease Study 2017 (GBD 2017) Results. Seattle, United States: Institute for Health Metrics and Evaluation (IHME); 2018.

[4] Ueda P, Woodward M, Lu Y, Hajifathalian K, Al-Wotayan R, Aguilar-Salinas CA, et al. Laboratory-based and office-based risk scores and charts to predict 10-year risk of cardiovascular disease in 182 countries: a pooled analysis of prospective cohorts and health surveys. Lancet Diabetes Endocrinol 2017;5:196-213. https://doi.org/10.1016/S2213-8587(17)30015-3.

[5] Sonnenberg FA, Beck JR. Markov Models in Medical Decision Making: A Practical Guide. Med Decis Making 1993;13:322-38. https://doi.org/10.1177/0272989X9301300409.

[6] Bellows BK, Ruiz-Negrón N, Bibbins-Domingo K, King JB, Pletcher MJ, Moran AE, et al. Clinic-Based Strategies to Reach United States Million Hearts 2022 Blood Pressure Control Goals: A Simulation Study. Circ Cardiovasc Qual Outcomes 2019;12:e005624. https://doi.org/10.1161/CIRCOUTCOMES.118.005624.

[7] Huffman MD, Rao KD, Pichon-Riviere A, Zhao D, Harikrishnan S, Ramaiya K, et al. A Cross-Sectional Study of the Microeconomic Impact of Cardiovascular Disease Hospitalization in Four Low- and MiddleIncome Countries. PLoS ONE 2011;6:e20821. https://doi.org/10.1371/journal.pone.0020821.

[8] Petty GW, Brown RD, Whisnant JP, Sicks JD, O'Fallon WM, Wiebers DO. Survival and recurrence after first cerebral infarction: A population-based study in Rochester, Minnesota, 1975 through 1989. Neurology 1998;50:208-16. https://doi.org/10.1212/WNL.50.1.208.

[9] Lin JK, Moran AE, Bibbins-Domingo K, Falase B, Pedroza Tobias A, Mandke CN, et al. Cost-effectiveness of a fixed-dose combination pill for secondary prevention of cardiovascular disease in China, India, Mexico, Nigeria, and South Africa: a modelling study. Lancet Glob Health 2019;7:e1346-58. https://doi.org/10.1016/S2214-109X(19)30339-0.

[10] Smolina K, Wright FL, Rayner M, Goldacre MJ. Long-Term Survival and Recurrence After Acute Myocardial Infarction in England, 2004 to 2010. Circ Cardiovasc Qual Outcomes 2012;5:532-40. https://doi.org/10.1161/CIRCOUTCOMES.111.964700.

[11] Law MR, Morris JK, Wald NJ. Use of blood pressure lowering drugs in the prevention of cardiovascular disease: meta-analysis of 147 randomised trials in the context of expectations from prospective epidemiological studies. BMJ 2009;338:b1665. https://doi.org/10.1136/bmj.b1665.

[12] India Hypertension Management Intiative. Punjab Hypertension Protocol n.d.

[13] Chow CK, Teo KK, Rangarajan S, Islam S, Gupta R, Avezum A, et al. Prevalence, Awareness, Treatment, and Control of Hypertension in Rural and Urban Communities in High-, Middle-, and Low-Income Countries. JAMA 2013;310:959-68. https://doi.org/10.1001/jama.2013.184182.

[14] Rau N, Nayak SK. Practical Guidelines for Hypertension Management. Med. Update 2013, Mumbai, India: The Association of Physicians of India; 2013, p. 69-73.

[15] Jaffe MG, Frieden TR, Campbell NRC, Matsushita K, Appel LJ, Lackland DT, et al. Recommended treatment protocols to improve management of hypertension globally: A statement by Resolve to Save Lives and the World Hypertension League (WHL). J Clin Hypertens 2018;20:829-36. https://doi.org/10.1111/jch.13280.

[16] Van Wijk BL, Klungel OH, Heerdink ER, de Boer A. Rate and determinants of 10-year persistence with antihypertensive drugs: J Hypertens 2005;23:2101-7. https://doi.org/10.1097/01.hjh.0000187261.40190.2e.

[17] Dennis T, Meera NK, Binny K, Sekhar MS, Kishore G, Sasidharan S. Medication adherence and associated barriers in hypertension management in India. CVD Prev Control 2011;6:9-13. https://doi.org/10.1016/j.cvdpc.2010.11.001.

[18] Cherry SB, Benner JS, Hussein MA, Tang SSK, Nichol MB. The Clinical and Economic Burden of Nonadherence with Antihypertensive and Lipid-Lowering Therapy in Hypertensive Patients. Value Health 2009;12:489-97. https://doi.org/10.1111/j.1524-4733.2008.00447.x.

[19] TSMSIDC. Medicines-Rate Contract. Telangana State Med Serv Infrastruct Dev Corp n.d. http://tsmsidc.telangana.gov.in/content.php?U=20\%20\&\&\%20T=Medicines-Rate\%20Contract (accessed January 7, 2020).

[20] WHO, Health Action International. Measuring price components. Meas. Med. Prices Availab. Affordabil. Price Compon. 2nd Ed., World Health Organization; n.d., p. 129-70.

[21] 1mg. About 1mg: India's leading digital consumer healthcare platform 2020. https://www.1mg.com/aboutUs (accessed May 15, 2020). 
[22] Prinja S, Chauhan AS, Bahuguna P, Selvaraj S, Muraleedharan VR, Sundararaman T. Cost of Delivering Secondary Healthcare Through the Public Sector in India. PharmacoEconomics 2020;4:249-61. https://doi.org/10.1007/s41669-019-00176-9.

[23] Central Government Health Scheme, GoI. Rate List for Hyderabad - CGHS Hyderabad w.e.f 17/11/2014. Hyderabad, India: Government of India; 2014.

[24] WHO. Country-specific inpatient and outpatient estimates in 2010 currency. Geneva: World Health Organization; 2010.

[25] Xavier D, Pais P, Devereaux P, Xie C, Prabhakaran D, Reddy KS, et al. Treatment and outcomes of acute coronary syndromes in India (CREATE): a prospective analysis of registry data. The Lancet 2008;371:1435-42. https://doi.org/10.1016/S0140-6736(08)60623-6.

[26] MSH. International Medical Products Price Guide. Arlington,Virginia: Management Sciences for Health; 2016.

[27] Husereau D, Drummond M, Petrou S, Carswell C, Moher D, Greenberg D, et al. Consolidated Health Economic Evaluation Reporting Standards (CHEERS) statement. BMJ 2013;346:f1049. https://doi.org/10.1136/bmj.f1049. 